\title{
Covid-19: Fully vaccinated people can carry as much delta virus as unvaccinated people, data indicate
}

\section{Shaun Griffin}

Adults who have been fully vaccinated against SARS-CoV-2 can carry the same viral load of the delta variant as those who are unvaccinated, a preliminary analysis of UK data suggests. ${ }^{1}$

The latest results from the UK's national covid-19 infection survey show that having two vaccine doses remains the most effective way to ensure protection against delta. But, although people who are fully vaccinated have a lower risk of becoming infected, those infected with the delta variant can carry similar virus levels as unvaccinated people, the data show. The authors said the implications for transmission were not yet clear but suggested that the potential for fully vaccinated individuals to transmit the virus to others would make achieving herd immunity more of a challenge.

Sarah Walker, professor of medical statistics and epidemiology at the University of Oxford and chief investigator of the survey, said, "We don't yet know how much transmission can happen from people who get covid-19 after being vaccinated-for example, they may have high levels of virus for shorter periods of time.

"But the fact that they can have high levels of virus suggests that people who aren't yet vaccinated may not be as protected from the delta variant as we hoped. This means it is essential for as many people as possible to get vaccinated-both in the UK and worldwide."

The analysis, published as a preprint and not yet peer reviewed, found that the Oxford-AstraZeneca and Pfizer-BioNTech vaccines both offered good protection against new infections but that performance was less good against delta than with the previously dominant alpha variant.

The researchers analysed 2580021 results from swabs taken from 384543 adults between 1 December 2020 and 16 May 2021, when the alpha variant predominated, and 811624 results from 358983 adults between 17 May and 1 August 2021, when delta prevailed. Two doses of either vaccine provided at least the same level of protection as that afforded through natural infection with the virus. For example, when delta prevailed, efficacy of the Pfizer vaccine was $80 \%$ (95\% confidence interval $77 \%$ to $83 \%$ ) at 14 or more days after two doses, higher than after two doses of the AstraZeneca vaccine (67\% (62\% to $71 \%)$ ) but not significantly different from the protection afforded by natural immunity in non-vaccinated people who had tested positive for SARS-CoV-2 (72\% (58\% to $82 \%)$ ).

The effectiveness of the two vaccines did not depend on the time between first and second doses. But people who were vaccinated after they had had covid-19 had more protection from vaccination than those who were vaccinated without a previous natural infection. For example, 14 days after a second AstraZeneca dose, on average the rates of all new covid-19 infections had dropped by $88 \%$ among those with prior infection, which compared with $68 \%$ in those without prior infection. The percentages were $93 \%$ and $85 \%$, respectively, for the Pfizer vaccine.

Speaking at a Science Media Centre briefing, Koen Pouwels, of Oxford University's Nuffield Department of Population Health and the report's lead author, emphasised the protective effect of vaccination in people with a high SARS-CoV-2 viral burden. He said that vaccine protection was $90 \%$ higher than in unvaccinated people one month after a second Pfizer vaccine, declining to $85 \%$ after two months and $78 \%$ after three months. For the AstraZeneca vaccine the equivalent protection was $67 \%, 65 \%$, and $61 \%$, respectively.

"Even with these slight declines in protection against all infections and infections with high viral burden, it's important to note that overall effectiveness is still very high, because we were starting at such a high level of protection," Pouwels said.

The study also showed that viral burden was lower immediately after two Pfizer doses but that it increased faster with time than after the AstraZeneca vaccine, leading to similar levels of viral burden around three months after second doses of both vaccines.

Commenting on the findings, the University of East Anglia's Paul Hunter noted, “There is now quite a lot of evidence that all vaccines are much better at reducing the risk of severe disease than they are at reducing the risk from infection. We now know that vaccination will not stop infection and transmission, [but it does] reduce the risk. The main value of immunisation is in reducing the risk of severe disease and death.”

Pouwels KB, Pritchard E, Matthews PC, et al, for the COVID-19 Infection Survey Team. Impact of delta on viral burden and vaccine effectiveness against new SARS-CoV-2 infections in the UK.

https://www.ndm.ox.ac.uk/covid-19/covid-19-infection-survey/results/newstudies.

This article is made freely available for use in accordance with BMJ's website terms and conditions for the duration of the covid-19 pandemic or until otherwise determined by BMJ. You may use, download and print the article for any lawful, non-commercial purpose (including text and data mining) provided that all copyright notices and trade marks are retained. 